\title{
Feasibility of "Hourglass-Pattern" Fascia Iliac Combined with Sacral Plexus Block in Elderly Femoral Neck Fractured Patients Undergoing Closed Reduction and Internal Fixation for Femoral Neck Fractures: A Prospective Observational Study
}

\author{
Qianyu $\mathrm{LI}^{1}$, Xiao Huang ${ }^{2}$ and Huiyue Wang ${ }^{3 *}$ \\ ${ }^{1}$ Master of Medicine, Anesthesia Department of Affiliated Hospital of Hebei University, China \\ ${ }^{2}$ Master of Medicine, Anesthesia Department of Beijing Chao-Yang Hospital, China \\ ${ }^{3}$ Master of Medicine, Department of Anesthesiology, No.1 Central hospital of Baoding, China
}

\begin{abstract}
Background: To evaluate the feasibility of "hourglass-sign" fascia iliac block and sacral plexus block for elderly femoral neck fractured patients with multiple complications.

Methods: A total of 32 patients with femoral neck fracture undergoing closed reduction and internal fixation for femoral neck fractures were included. Ultrasound-guided "hourglass-sign" fascia iliac block and sacral plexus block anesthesia were applied to all patients. The success rate of anesthesia, the hemodynamic parameters, VAS scores, general clinical data and perioperative information would be collected.

Results: 30 patients were included and analyzed into the study finally, all patients were diagnosed with different systemic diseases before surgery. The successful rate of "hourglass-sign" fascia iliac block combined with sacral plexus block guided by ultrasound and nerve stimulator was $93.8 \%$. The change of MAP at different time points was about $5 \%$ of the baseline values, and the heart rate was about $7 \%$. VAS scores decreased significantly after anesthesia and continued at least 1 hour after surgery. The anesthesia evaluation degree of most patients was satisfactory. There were no complications or adverse reactions associated with anesthesia.
\end{abstract}

Conclusion: This combined nerve block provided effective anesthesia for closed reduction and internal fixation for femoral neck fractures of elderly patients, rarely caused instability in the circulatory system, had fewer adverse reactions and could be widely used in clinical practice.

Trial Registry: This trial was registered at Chinese clinical trial (ChiCTR2000034114)

ABBREVIATIONS: ASA: American Society of Anesthesiologists; BMI: Body Mass Index; ECG: Electrocardiogram; NIBP: Non-Invasive Blood Pressure Monitoring; NSAIDs: Nonsteroidal Anti-Inflammatory Drugs; VAS: Visual Analogue Scale; MAP: Mean Arterial Pressure

Quick Response Code:

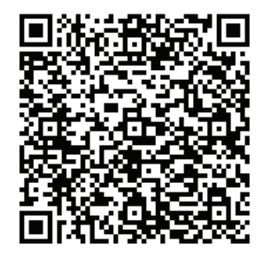

Address for correspondence: Huiyue Wang, Master of Medicine, Department of Anesthesiology, No.1 Central hospital of Baoding, China

Received: March 26, $2021 \quad$ Published: April 05, 2021

How to cite this article: Qianyu L, Xiao H, Huiyue W. Feasibility of "Hourglass-Pattern" Fascia Iliac Combined with Sacral Plexus Block in Elderly Femoral Neck Fractured Patients Undergoing Closed Reduction and Internal Fixation for Femoral Neck Fractures: A Prospective Observational Study. 2021- 3(2) OAJBS.ID.000268. DOI: 10.38125/OAJBS.000268 


\section{INTRODUCTION}

The number of elderly patients with hip fracture is increasing progressively which has become a global public health problem. Femoral neck fracture is a common type of hip fracture developing in the elderly and accounts for about $50 \%$ of hip fracture [1]. Surgery to treat femoral neck fracture was associated with a better prognosis outcome [2]. Thus, most of these patients need surgical treatment at the early stage of trauma. At present, closed reduction and internal fixation for femoral neck fractures is one of the most accepted surgical orthopedic procedures for these patients [3]. Elderly patients with femoral neck fracture are easy to complicate with a variety of basic diseases, such as hypertension, diabetes, cardiopulmonary diseases, etc. At the same time, they generally had a history of anticoagulant drug therapy. The choice of anesthesia for these patients is not limited to traditional methods. Peripheral nerve blocks have definite advantages over general anesthesia and intrathecal anesthesia as high safety, clear effect, have little physiological disturbance and decrease the incidence of postoperative complications and mortality. Furthermore, nerve blocks can provide sufficient perioperative analgesia, enable the patients to through the perioperative period smoothly and get out of bed early for conventional activities. In recent years, it has been increasingly reported on the use of old patients with multiple complications [4]. Ultrasound-guided peripheral nerve block has been extensively used in clinical practice over the years [5,6]. Due to its small impact on patients' hemodynamics, cognitive function and high security, it is especially suitable for elderly and critically ill patients [7].

Fascia iliac block is widely used to provide anesthesia and analgesia in hip replacement surgery. Evidence suggests that "hourglass-sign" fascia iliac block can effectively block the main branches of the lower limbs innervated by the lumbar plexus including the obturator nerve [8,9]. "Hourglass-sign" combined with sacral plexus block can theoretically meet the criteria of closed reduction and internal fixation for femoral neck fractures. Several studies have reported that ultrasound-guided nerve block anesthesia had been successfully applied in hip surgery [10], but the safety and effectiveness of lumbar plexus block and fascia iliac block for old patients in the clinic are still controversial $[11,12]$. At present, there is no relevant report on the combination of "hourglass-sign" and sacral plexus block in elderly patients with femoral neck fracture undergoing closed reduction and internal fixation for femoral neck fractures. Therefore, it is necessary to find a minimally invasive, effective and innovative anesthesia technique. The purpose of this study is to evaluate its effect and provide a basis for elderly femoral neck fractured patients with multiple complications.

\section{MATERIAL AND METHODS}

\section{Trial Design and Oversight}

This prospective, observational study was registered at Chinese clinical trial (ChiCTR2000034114) on June 24, 2020. After obtaining approval of the ethics committee of No.1 central hospital of Baoding, Hebei ((No. 2019083), and written informed consent, we recruited patients scheduled for closed reduction and internal fixation surgery from June 25, 2020 to September 1, 2020. We intended to enroll 32 participants and the study informed written consent had been obtained from all participants before enrollment to the study. The inclusion criteria of the patients were: femoral neck fractured patients planning to receive closed reduction and internal fixation for femoral neck fractures; aged 65 85 years, American Society of Anesthesiologists (ASA) II III, body mass index (BMI) $18 \sim 30 \mathrm{~kg} / \mathrm{m}^{2}$. The following patients were excluded: patients who were allergic to amide local anesthetics; with the central nervous system or peripheral nerve diseases; with current chronic analgesic therapy; having a history of opioid dependence; confused thinking and unable to communicate well and cooperate with investigators; required postoperative mechanical ventilation; receiving anticoagulation, experiencing any bleeding disorder or had extreme intra or postoperative bleeding risk.

\section{Trial Procedures}

After entering the room, all patients received oxygen supply with oxygen masks and venous access was established. Routine preoperative assessments were performed for all patients. All patients were monitored with 5 leads electrocardiogram (ECG), non-invasive blood pressure monitoring (NIBP) and pulse oximetry. Radial artery puncture and catheterization with local anesthesia were performed to monitor invasive blood pressure. The combined nerve block was performed by an experienced consultant anesthesiologist. Ultrasound-guided "hourglass-sign" fascia iliac block in the supine position was prior. A two - dimensional portable ultrasonic instrument was used for positioning, and the high-frequency 5-10 MHz linear array ultrasound transducer was vertically positioned in third of the middle and lateral inguinal ligament. Local anesthesia was injected after identifying "hourglass-sign" and the anatomy of the fascia iliac. Then a 100 $\mathrm{mm}, 21 \mathrm{G}$ needle was inserted from the caudal to cranial parallel to the probe with the technique of hydro-dissection, and $40 \mathrm{ml}$ ropivacaine in concentrations of $0.33 \%$ was injected slowly after confirming the correct location. "Hourglass-sign" fascia iliac block worked through a pinprick test 3-5 minutes later and sacral plexus block was performed next. Patients were placed in a lateral position with the surgical side uppermost, and the low-frequency $2-5 \mathrm{MHz}$ ultrasound transducer was placed in half of the distance from the greater trochanter of the femur and the posterior superior iliac spine. A needle connected to a nerve stimulator with a current of $1 \mathrm{~mA}$ and frequency of $2 \mathrm{~Hz}$ was inserted near to the sacral plexus parallel to the probe. When foot plantarflexion or dorsiflexion was elicited, the stimulation current was reduced to $0.4 \sim 0.5 \mathrm{~mA} .20 \mathrm{~mL}$ $0.375 \%$ ropivacaine was injected incrementally after verifying the needle tip position with the technique of hydro-dissection.

Another anesthesiologist who was not involved in the procedure was responsible for the evaluation of the anesthetic effect and recorded it. The patient would receive general anesthesia if the anesthetic doesn't work 30 minutes after the implementation of the nerve block operation. Norepinephrine and atropine would be used to regulate heart rate and blood pressure according to monitoring data. Dexmedetomidine was continuously pumped at a rate of $0.5 \mu \mathrm{g} . \mathrm{kg}-1 . \mathrm{h}-1$.

\section{Data Collection}

Multi-mode analgesia was carried out postoperatively with nonsteroidal anti-inflammatory drugs (NSAIDs) combined with an intramuscular injection of dezocine. Visual analogue scale (VAS) was explained in detail to each patient by a professional researcher. Where 0 scores as no pain at all and 10 as the worst imaginable pain. The primary outcome was the success rate of anesthesia. In this study, anesthesia was considered successful in cases that the operation was completed successfully with the combined nerve block anesthesia. The case who could not receive operation 
smoothly for the poor motor block or sensory block would change to general anesthesia and would be considered as anesthesia failure. Secondary outcomes were the measurement of the mean arterial pressure (MAP) and heart rate six-time points: 5 min before anesthesia $\left(\mathrm{t}_{0}\right)$, placing position after effective iliac fascia block $\left(t_{1}\right), 10$ min after anesthesia $\left(t_{2}\right)$, beginning of the operation $\left(t_{3}\right)$, 30 min after surgery $\left(t_{4}\right)$ and 1 hour after surgery $\left(t_{5}\right)$; VAS scores at ten-time points: $t_{0}-t_{5}, 6$ hours after surgery $\left(t_{6}\right), 12$ hours after surgery $\left(\mathrm{t}_{7}\right), 24$ hours after surgery $\left(\mathrm{t}_{8}\right)$ and 48 hours after surgery $\left(t_{9}\right)$. Besides, general clinical data and intraoperative information, length of surgery and hospital stay, the time from the end of the surgery to engaged in activities the incidence of anesthesia-related complications and adverse reactions would be collected.

\section{Statistical Analysis}

Statistical analysis was performed using the Prism 7.0. Data were analyzed by SPSS for Windows (version 25.0, International
Business Machines Corporation, USA). Continuous, normally distributed data were expressed as mean \pm standard deviation unless otherwise stated. Categorical data were expressed as numbers except for special statement. $P$ values of $<0.05$ were considered statistically significant.

\section{RESULTS}

Two patients were changed to general anesthesia for the failure of the nerve block. A total of 30 patients were included and analyzed in the study finally. All patients were elderly, with an average age of 76 years. Almost half of the patients were overweight. All patients were diagnosed with different systemic diseases before surgery. (Table 1) Intraoperative and postoperative variables were showed in Table 2. The anesthesia evaluation degree of most patients was satisfactory. There were no complications or adverse reactions associated with anesthesia.

Table 1: Preoperative patient characteristics.

\begin{tabular}{|c|c|}
\hline Variables & $\mathbf{N}=\mathbf{3 0}$ \\
\hline Age (years) & $76.9 \pm 4.3$ \\
\hline Sex-female; $n(\%)$ & $17(56.7)$ \\
\hline Body mass index $\left(\mathrm{kg} / \mathrm{m}^{2}\right)$ & $25.7 \pm 1.9$ \\
\hline Diabetes; $n(\%)$ & $19(63.3)$ \\
\hline Hypertension; $n(\%)$ & $23(76.7)$ \\
\hline Coronary artery disease; $n(\%)$ & $9(30.0)$ \\
\hline Lung disease; $n(\%)$ & $10(33.3)$ \\
\hline ASA classification; $n(\%)$ & $23(76.7)$ \\
\hline Class II & $7(23.3)$ \\
\hline Class III & \\
\hline
\end{tabular}

Note: The data were presented as means \pm standard deviation for continuous variables and as percentages (\%) for categorical variables ASA: American Society of Anesthesiologists

Table 2: Intraoperative and postoperative variables.

\begin{tabular}{|c|c|}
\hline Variables & $\mathbf{N}=\mathbf{3 0}$ \\
\hline Noradrenaline; $n(\%)$ & $2(6.7)$ \\
\hline Atropine; $n(\%)$ & $2(6.7)$ \\
\hline Volume of liquid infusion $(\mathrm{mL})$ & $1156.7 \pm 104.0$ \\
\hline Intraoperative bleeding volume $(\mathrm{mL})$ & $121.3 \pm 39.1$ \\
\hline Intraoperative urinary volume $(\mathrm{mL})$ & $267.0 \pm 71.3$ \\
\hline Length of surgery $(\mathrm{h})$ & $1.4 \pm 0.2$ \\
\hline The time from the end of the surgery to engaged in activities $(\mathrm{h})$ & $16.0 \pm 1.9$ \\
\hline Length of hospital stay $(\mathrm{d})$ & $5.8 \pm 0.7$ \\
\hline
\end{tabular}

Example of images are illustrated in Figure 1 and Figure 2. In half of the patients, the image quality was graded as average and nerve stimulation was successful in validating the image of "hourglasssign" and sacral plexus. The heart rates and MAPs at different time points were showed in Table 3. The change of MAP at different time points was about $5 \%$ of the baseline values, and the heart rate was about $7 \%$. Heart rate and blood pressure did not fluctuate greatly at different time points and persist relatively stable.

From Figure 3, the VAS score showed statistically significant changes along the $\mathrm{t} 1-\mathrm{t} 9$ when compare with the baseline $(P<0.05)$. VAS scores decreased significantly after anesthesia until 6 hours after surgery, then began to recover from the lowest value. The average VAS score two days after surgery was 3.5 points, which was still significantly lower than the VAS of baseline $(P<0.05)$.

\section{DISCUSSION}

In the present study, we applied "hourglass-sign" fascia iliac block combined with sacral plexus block guided by ultrasound to those with severe circulatory deficiency, and observed if it could meet the surgical need, the effects on hemodynamic fluctuations, as well as the rate of postoperative complications. The results showed that the innovative anesthesia method could provide perfect perioperative analgesia, met the surgical needs, resulted in little intraoperative circulation fluctuation and no adverse reactions. 


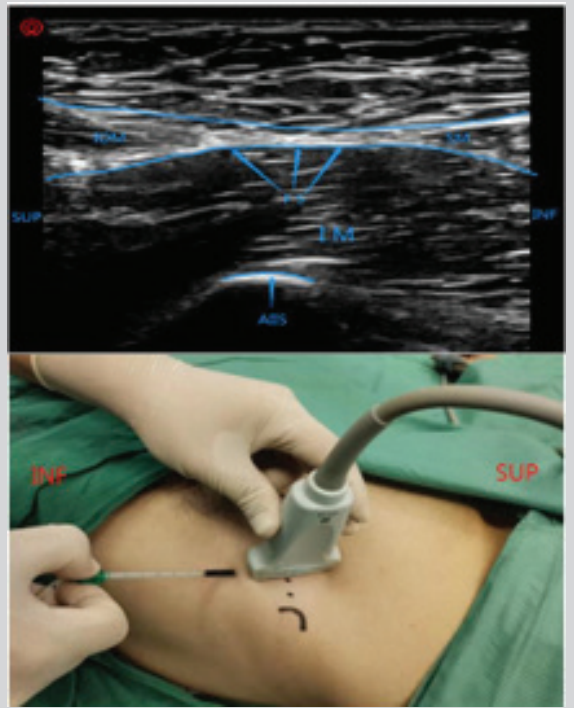

Figure 1: Ultrasonography of hourglass-pattern, the positions of transducer and the puncture point.

Note: AllS: Anterior Inferior lliac Spine; SUP: Superior; INF: Inferior; FS: Fascia lliaca; IM: lliopsoas Muscle; IOM: Internus Obiquus Abdominis Muscle; SM: Sartorius Muscle

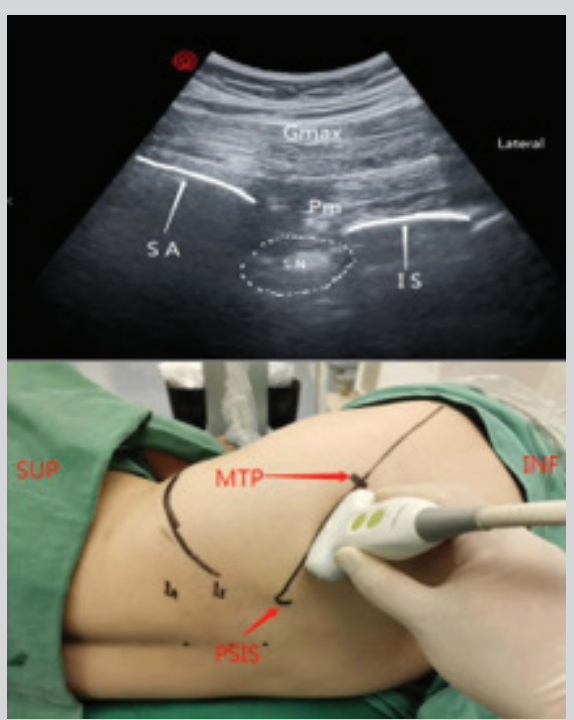

Figure 2: Ultrasonography of sacral plexus, the positions of transducer and the puncture point of sacral plexus block.

Note: PSIS: Posterior Superior lliac Spine; SUP: Superior; INF: Inferior; MTP: Midpoint of the Line Between Greater Trochanter of Femur and the PSIS; Gmax: Gluteus Maximus Muscle; PM: Piriformis Muscle; SN: Sacral Plexus; SA: Sacrum; IS: Ischium

Table 3: MAP and HR at different time points.

\begin{tabular}{|c|c|c|}
\hline Time & MAP & HR \\
\hline$t_{0}$ & $103.5 \pm 8.4$ & $80.6 \pm 6.3$ \\
\hline$t_{1}$ & $106.9 \pm 7.9$ & $75.7 \pm 6.2$ \\
\hline$t_{2}$ & $102.1 \pm 8.1$ & $73.5 \pm 15.1$ \\
\hline$t_{3}$ & $102.0 \pm 7.5$ & $74.2 \pm 6.0$ \\
\hline$t_{4}$ & $99.7 \pm 6.7$ & $73.2 \pm 6.1$ \\
\hline$t_{5}$ & $98.8 \pm 6.7$ & \\
\hline
\end{tabular}

Note: The data were presented as means \pm standard deviation. MAP: Mean Arterial Pressure. 


\section{Perioperative Visual Analogue Scale (VAS)}

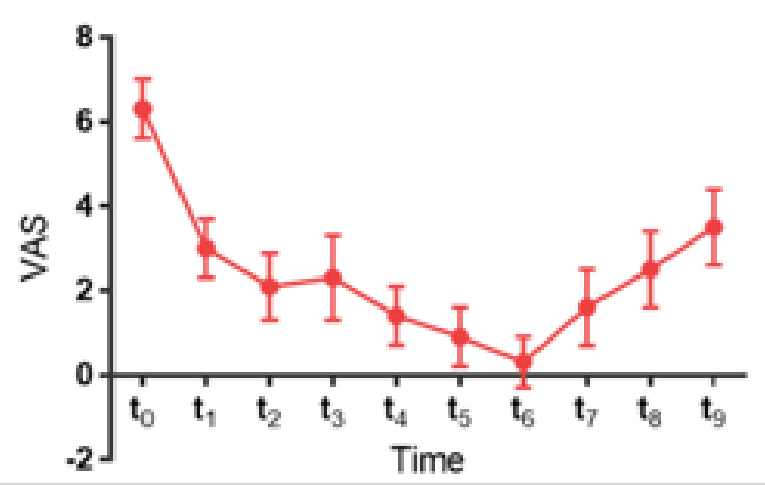

Figure 3: The level of VAS at different time points. Values are presented as the mean \pm SD. Highest scores indicate the worst pain intensity. $t_{0}=5 \mathrm{~min}$ before anesthesia, $t_{1}=$ placing position after effective iliac fascia block, $t_{2}=10 \mathrm{~min}$ after anesthesia, $t_{3}=$ beginning of the operation, $t_{4}=30$ min after surgery, $t_{5}=1$ hour after surgery, $t_{6}=6$ hours after surgery, $t_{7}=12$ hours after surgery, $t_{8}=24$ hours after surgery, $t_{9}=48$ hours after surgery.

For closed reduction and internal fixation for femoral neck fractures, the perfect block of the femoral nerve and lateral femoral cutaneous nerve is essential, and the obturator nerve block is also crucial to relieve the pain during the process of fracture traction and reduction. Vermeylen et al. [17] had shown the "hourglasssign" fascia iliac block can effectively block the main branches of the lumbar plexus including the obturator nerve and lateral femoral cutaneous nerve compared with the widely used fascia iliac block method. According to Hebbard [18], the blocking rate of the femoral nerve and lateral femoral cutaneous nerve can even up to $100 \%$. The sacral plexus is composed of L4-5 of the lumbosacral trunk, the anterior branches of all sacral and caudal nerves. The sacral plexus is located in the pelvic cavity posterior part of the internal iliac artery in front of the sacrum and piriform muscle. The branches of the sacral plexus distributed in the pelvic wall, buttock, perineum, posterior thigh, leg, foot muscle and skin and many short muscular branches of it innervate piriformis, obturator muscle and quadratus femoris. Therefore, ultrasound and nerve stimulator guided "hourglass-sign" fascia iliac block combined with sacral plexus block can block almost the whole area of buttock and limb and satisfy the anesthetic requirements for that surgery. The results of this study indicate that the implementation of "hourglasssign" fascia iliac block combined with sacral plexus block is a simple, safe and the success anesthetic technique, especially for the elderly patients who are vulnerable to suffer from serious perioperative complications with general anesthesia. Nerve blocks have fewer complications, such as hematoma at the puncture location and hemodynamic fluctuation.

Furthermore, since peripheral nerve block does not influence pelvic and abdominal visceral nerves, the patients will not have the risk of urinary retention, spinal headache, nausea or vomiting postoperatively. Also, there is no need for postoperative fasting and spinal headache risk compared with general anesthesia and intraspinal anesthesia.

In the present study, two cases were ruled out due to the failure of nerve block anesthesia. One switch to general anesthesia because he could not tolerate the pain in lateral position when performing sacral plexus block, and the other was due to the lack of a definite anesthetic effect. The possible reason is that the large external rotation angle of some patients with femoral neck fracture leads to unclear ultrasonography of "hourglass-sign" fascia iliac. So local anesthetics were not completely and effectively injected into the correct space.

Pain in the hip fracture can aggravate stress response in patients preoperatively [19]. When anesthesia is performed in a lateral position, it will inevitably cause pain and the risk of serious cardiovascular accidents in elderly patients with hip fracture. In the present study, "hourglass-sign" fascia iliac was perform for patients in a supine position firstly, which can effectively relieve the pain caused by performing sacral plexus block in the lateral position, therefore avoiding the need to move them while they were still in pain by cause of their fracture. According to our results, the anesthetic method used in this study as an innovative way has less impact on elderly patients with multiple coexisting diseases. It can reduce the risk of poor prognosis caused by intraoperative circulatory instability [20]. And the VAS score at each time point showed that the improved anesthesia method can provide perfect perioperative analgesia. The early postoperative activity was achievable in all patients, which may be related to the low concentration of ropivacaine and its characteristic of separation between motion and sensation and is less potent for motor block [21]. What has been mentioned above are all conducive to the early postoperative rehabilitation of elderly patients and complies to the concept of ERAS [22].

\section{LIMITATIONS}

Although our prospective observational study has proved that the combination of "hourglass-sign" fascia iliac block and sacral plexus block could bring many benefits to the elderly patients with a femoral neck fracture, there were several limitations to this study. Firstly, Due to the small sample size, a relatively small number of complications came up in the timeframe study and lacking a control group, the capacity to evaluate nerve block related complications was limited.

Secondly, a single-center study maybe with potential for selection bias. What's more, the effective and safe concentration and volume of this combined nerve block are still uncertain. The results in our study might provide a basis for further trials to assess the safety and effectiveness of this anesthesia technique. It's urgent to need more multicentre, large-scale and high-quality research in the future. 


\section{CONCLUSION}

In summary, the success rate of "hourglass-sign" fascia iliac block combined with sacral plexus block guided by ultrasound and nerve stimulator was high enough. This technique produced clinical evidence of blocking. It could provide effective anesthesia for closed reduction and internal fixation for femoral neck fractures of elderly patients, rarely caused instability in the circulatory system and had fewer adverse reactions. This compound nerve block is advisable for old patients with serious cardiopulmonary deficiency or unstable circulation and could be widely used in clinical practice.

\section{AUTHORS CONTRIBUTION}

Conception and design, critical revision for this study protocol: Huiyue Wang and Qianyu Li. Recruiting subjects: Anshi Wu. Anesthesia treatment: Huiyue Wang. Data collection: Huiyue Wang and Qianyu Li. Data analysis and interpretation of data: Xiao Huang. All authors read and approved the final manuscript. The authors declare that they have no conflict of interest. Huiyue Wang and Xiao Huang contributed equally to this work.

\section{FUNDING}

This study was funded by Baoding Science and technology planning project (no. 1951ZF066). Role of the Funder/Sponsor: The funders had no role in the design and conduct of the study; collection, management, analysis, and interpretation of the data; preparation, review, or approval of the manuscript; and decision to submit the manuscript for publication.

\section{REFERENCES}

1. Zajonz D, Behrens J, Brand AG (2019) Femoral neck fractures in old age treated with hemiarthroplasty: Effects of preoperative waiting time and timing of surgery on the risk of periprosthetic infections. Orthopade 48(3): 224-231.

2. Wang Z, Bhattacharyya T (2017) Outcomes of hemiarthroplasty and total hip arthroplasty for femoral neck fracture: A medicare cohort study. J Orthop Trauma 31(5): 260-263.

3. Zhang LL, Zhang Y, Ma X, Youwen L (2017) Multiple cannulated screws vs. dynamic hip screws for femoral neck fractures: A meta-analysis. Multiple kanülierte Schrauben vs. dynamische Hüftschrauben bei Femurhalsfrakturen: Eine Metaanalyse. Orthopade 46(11): 954-962.

4. Pawa A, Wight J, Onwochei DN, R Vargulescu, I Reed, et al. (2018) Combined thoracic paravertebral and pectoral nerve blocks for breast surgery under sedation: a prospective observational case series. Anaesthesia 73(4): 438-443.

5. Singh H, Jones D (2013) Hourglass-pattern recognition simplifies fascia iliaca compartment block. Reg Anesth Pain Med 38(5): 467-468.

6. Venkataraju A, Narayanan M, Phillips S (2020) Parasacral ischial plane (PIP) block: An easy approach to sacral plexus. J Clin Anesth 59: 103105.

7. Mei B, Lu Y, Liu X, Zhang Y, Gu E, et al. (2019) Ultrasound-guided lumbar selective nerve root block plus T12 paravertebral and sacral plexus block for hip and knee arthroplasty: Three case reports. Medicine (Baltimore) 98(22): e15887.
8. Zhang H, Miao Y, Qu Z (2019) Supra-inguinal injection for fascia iliaca compartment block results in more consistent spread towards the lumbar plexus than an infra-inguinal injection: a volunteer study: a concern on the influence of patient's breathing. Reg Anesth Pain Med $44(10)$.

9. Zhang XY, Ma JB (2019) The efficacy of fascia iliaca compartment block for pain control after total hip arthroplasty: A meta-analysis. J Orthop Surg Res 14(1): 33

10. Chen L, Liu J, Yang J, Zhang Y, Liu Y, et al. (2016) Combined fascia iliaca and sciatic nerve block for hip surgery in the presence of severe ankylosing spondylitis: A case-based literature review. Reg Anesth Pain Med 41(2): 158-163.

11. Gamli M, Sacan O, Baskan S, Nermin G (2011) Combined lumbar plexus and sciatic nerve block for hip fracture surgery in a patient with severe aortic stenosis. J Anesth 25(5): 784-785.

12. Shariat AN, Hadzic A, Xu D, Shastri U, Kwofie K, et al. (2013) Fascia iliaca block for analgesia after hip arthroplasty: a randomized double-blind, placebo-controlled trial. Reg Anesth Pain Med 38(3): 201-205.

13. Simunovic N, Devereaux PJ, Sprague S, Gordon HG, Emil Schemitsch, et al. (2010) Effect of early surgery after hip fracture on mortality and complications: systematic review and meta-analysis. CMAJ 182(15): 1609-1616.

14. Desai V, Chan PH, Prentice HA, Zohman GL, Diekmann GR, et al. (2018) Is anesthesia technique associated with a higher risk of mortality or complications within 90 days of surgery for geriatric patients with hip fractures? Clin Orthop Relat Res 476(6): 1178-1188.

15. Berninger MT, Friederichs J, Leidinger W (2018) Effect of local infiltration analgesia, peripheral nerve blocks, general and spinal anesthesia on early functional recovery and pain control in total knee arthroplasty. BMC Musculoskelet Disord 19(1): 232

16. Grossi P, Urmey WF (2003) Peripheral nerve blocks for anaesthesia and postoperative analgesia. Curr Opin Anaesthesiol 16(5): 493-501.

17. Vermeylen K, Desmet M, Leunen I (2019) Supra-inguinal injection for fascia iliaca compartment block results in more consistent spread towards the lumbar plexus than an infra-inguinal injection: a volunteer study. Reg Anesth Pain Med 44(4).

18. Hebbard P, Ivanusic J, Sha S (2011) Ultrasound-guided supra-inguinal fascia iliac block: a cadaveric evaluation of a novel approach. Anaesthesia 66(4): 300-305.

19. Beaudoin FL, Haran JP, Liebmann O (2013) A comparison of ultrasoundguided three-in-one femoral nerve block versus parenteral opioids alone for analgesia in emergency department patients with hip fractures: a randomized controlled trial. Acad Emerg Med 20(6): 584-591.

20. Monk TG, Bronsert MR, Henderson WG, Michael PM, John SP, et al (2015) Association between intraoperative hypotension and hypertension and 30-day postoperative mortality in noncardiac surgery [published correction appears in Anesthesiology. Anesthesiology 123(2): 307-319.

21. Camorcia M, Capogna G, Lyons G, Columb M (2004) Epidural test dose with levobupivacaine and ropivacaine: determination of ED (50) motor block after spinal administration. Br J Anaesth 92(6): 850-853.

22. Kehlet H, Dahl JB (2003) Anaesthesia, surgery, and challenges in postoperative recovery. Lancet 362(9399): 1921-1928. 